\title{
Implementation of Earned Value Management tools in the Wendelstein 7-X Project
}

\author{
A.Lorenz, H.-S.Bosch, K. Küttler \\ Max-Planck-Institute für Plasma Physik, Euratom Association, \\ D-17491 Greifswald, Germany \\ axel.lorenz@ipp.mpg.de
}

\begin{abstract}
The stellarator project W7-X, currently under construction, has recently implemented several Earned Value Management tools to enable a tighter monitoring of internal processes such as the $W 7-X$ assembly process, the diagnostic engineering and the manufacture of in-vessel components. Specification and implementation of these tools has posed several challenges characteristic of large scale research projects that are subject to a number of changes during their life cycle. After putting these tools in operation, they have proven as early and transparent performance indicators for project control.
\end{abstract}

Keywords- project control, earned value management; assembly; diagnostic engineering; in-vessel components

\section{INTRODUCTION}

Earned Value Management (EVM) is now an accepted method for measuring and controlling a project's progress. The EVM method as described in [1], has found increasing use in both, industry and government sponsored projects. It is especially valuable in projects where progress is primarily achieved by the application of personnel resources, as e.g. in IT projects. The motivation for introducing EVM arises because project tracking often separates schedule monitoring from cost analysis. Often work breakdown structures (WBS) are followed by milestone trend analysis and costs are compared against planned budgets in separate controlling tools. Also, in some organizations individual management procedures are followed for schedule and for cost monitoring. In many cases this approach is reasonable and sufficient, however, in R\&D projects, progress is rarely proportional to budget usage or time. The criticality of a budget overrun is well known, however, project success is in some cases just as endangered if the project underspends, while progress seems to be in line with the schedule. The latter refers predominantly to early project stages featuring calculations, tests and development work, and conceptual designs, where schedules are often unstable and assessed by the individual judgment of the Responsible Officer. Monitoring progress and cost together against time, taking into account such a nonlinear dependence, is possible only with an Earned Value Analysis [1].

In general, EVM requires a detailed planning of the project which expresses all the planned work packages with their respective cost/value as a function of time, the so-called Planned Value (PV). The final value of this parameter, the
Budget at Completion (BAC), is the planned total cost of the project. During project execution, in pre-determined intervals, the actual acquired costs, Actual Cost (AC) and the Earned Value (EV) are determined and compared to the PV for the respecticve point in time. The Earned Value is the sum of the planned costs for all work packages finished at that moment.

Comparison of the three parameters PV, EV and AC in a standard analysis graph clearly describes the status of a project and even allows measuring this status [1]. In principle, EVM also has the capability to extrapolate the data to determine an estimate for the time at completion, TAC, and cost at completion, but such extrapolations are not used in our project.

In this paper, the particular goals, the special set-up and first results of three EVM based monitoring tools implemented in different areas of the Wendelstein 7 -X project are presented.

\section{EVM TOOL For ASSEMBLY}

\section{A. Goal}

The assembly of W7-X follows a tight schedule using extensive human and financial resources in a complex network of work packages, different assembly stands and various differing requirements on the personnel qualifications. Under these conditions comprehensive monitoring of the work progress against the assembly planning is extremely important, since even small relative deviations from the plan carry large absolute cost increments. Consequently, the tool was mainly introduced as an early warning system for possible budget overruns, complementary to the existing tight schedule monitoring of the assembly processes.

\section{B. Set-Up}

The set-up of the assembly tool has been based on the existing baseline plan derived from the bottom up, resource weighted assembly planning of all assembly steps. The plan aggregates singular assembly activities of the order of single man hours into measurable work packages to be completed over a number of weeks. In this way, the „weighted milestone” method [1] can directly be used to define the PV for such work packages. The size of these work packages has been defined in a way that their fulfillement can clearly be judged and an EV either of $0 \%$ or $100 \%$ can be attributed to each work package.

Technically, the Wendelstein $7-\mathrm{X}$ assembly plan is structured into 6 assembly "units" (the assembly of 5 identical 
modules and the "torus assembly", i.e. connection of the modules to a torus and the installation of peripheral systems), each of them characterized by their own PV-curve. Fig. 1 shows the PV for each unit and the cumulated curve for all 6 assembly units covering the period from the start of monitoring in 2010 until the planned end of assembly in 2014 as baselined in 2010. A time resolution of 1 month was chosen not only as a compromise between efficient data collection and the required resolution for earned value analysis, but also because it fits very well the rhythm of monthly billing for the external work forces which are responsible for the majority of the assembly man power. Since the tool has been set up only after the start of W7-X assembly, this concept and the implementation have required compromises regarding the allocation of $\mathrm{AC}$ to the assembly rigs and work packages. Specifically, a detailed allocation of the costs of single workers in a specific work package had to be abandoned as it would now be too laborious and costly a system for monitoring the man power allocation and for the AC readout in the existing SAP software. Instead, the Actual Costs for a given month is distributed to the assembly "units" proportional to the planned values of this "unit" for the respective month. For the analysis of the sum of all assembly "units" this does not pose a problem, however, for single unit monitoring, the AC allocated to this unit has some uncertainty, thereby decreasing the informative value of the EVM charts for single "units".

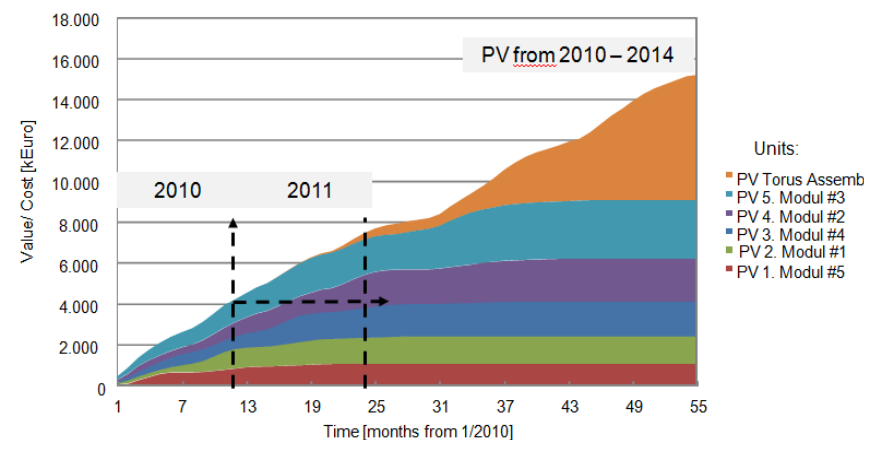

Figure 1. Planned Value for EVM tool of W7-X assembly cumulating assembly of 5 identical modules and assembly of torus and installation of peripheral systems

\section{Results}

Figure 2 shows the EVM line charts for December 2010 (2a) and for May 2011 (2b) for the sum of all assembly "units" as described above. As can be seen in Fig. 2a, at the end of 2010 the achieved EV matched PV exactly catching up on intermediate delays of up to 6 weeks during the course of the year. The schedule delays were mostly due to technical issues during the start of the very complex port assembly processes. Positively, AC remained some $300 \mathrm{k} €$ below the projected budget. The additional expenses of having to inject more personnel in mid 2010 to catch up on schedule delays did not lead to a budget overrun.

The yearly review of assembly schedule and assembly procedures at the end of 2010 has lead to a base-line plan adjustment, resulting in a revised $2011 \mathrm{PV}$ trajectory shown in Fig. 2b. The PV difference is illustrated by the addition of the
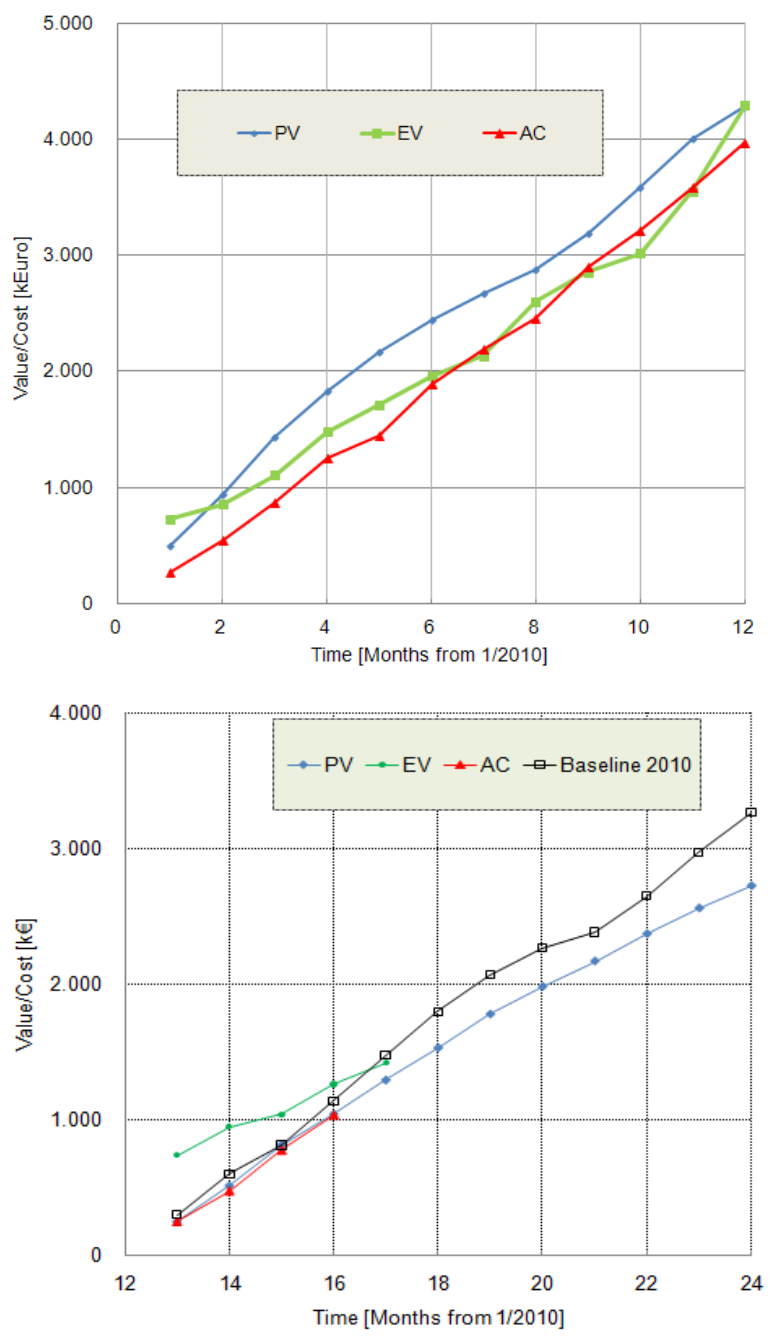

Figure 2. a (top): EVM chart for W7-X assembly in 2010, schedule delays identified by comparing horizontal gap between EV and PV and cost performance by comparing EV to AC. Fig. 2b (bottom): EVM chart for W7-X assembly for May 2011 after a change of the baseline plan at end of 2010.

PV curve titled "Baseline 2010". Running for about 3 months almost on the same trajectory as the 2011 PV curve the old plan suggested an increased PV gradient ending 2011 some $500 \mathrm{k} €$ above the 2011 PV curve. This budget cut does not simply reflect the savings observed in 2010, but is the result of a revised assessment of the assembly resource requirements and optimization of some assembly procedures without compromising the schedule. As can also be seen in Fig. 2b, EV measurements of the first few months in 2011 showed a strong performance, thought to be due to repetition of the port assembly steps, now on the second (\#1) and the third (\#4) module of Wendelstein 7-X [2]. Fig. 2b shows the status of end of May 2011, when the Earned Value for May has been updated already. The Actual Costs accumulated in May, however, have been attributed to April to incorporate the fact that the accounting of the external manpower usually takes place with one month delay. 


\section{EVM TOOL For DIAGNOSTIC ENGINEERING}

\section{A. Goal}

The tool for the diagnostic engineering works on a much shorter time scale by tracking the progress of each diagnostic design against the design hours spent. The preparation of the first 25 W7-X diagnostics has entered an intensive phase of engineering design in order to start timely manufacturing. Permanent knowledge of how much progress is being made at this stage is vital for an effective project control. Consequently, the Planned Value for the diagnostic engineering has been set up with a higher time resolution of one week.

\section{B. Set-Up}

$\mathrm{PV}$ is not only determined for each diagnostic project, but also is considered as the sum of several diagnostics grouped by category such as in-vessel or operation relevant diagnostics, for coherent priority control. The challenge of this tool is the sharp definition of the design steps to allow for transparent status evaluation. Therefore each design step, i.e. a work package (typical size of $40-200$ hours) is identified and agreed upon after iterations between the physics and engineering departments. Calculation of PV is simplified by considering only one or two designers per diagnostic. Assuming that a designer cannot dedicate $100 \%$ of his time to pure design work, a $33 \mathrm{~h}$ design time per week (out of $40 \mathrm{~h}$ ) has been chosen as a first guideline. A flexible tool set up, however, will allow for easy adjustment should systematic deviations between PV and AC occur.

The EV assessment of a project design is carried out monthly by the designer's department heads who were before involved in the original estimate of the design tasks. Unlike in the assembly EVM tool earned value is credit for progress of $0 \%, 50 \%, 75 \%$ and $100 \%$. This type of rating has been chosen to account for the frequent switch between design tasks leading in some cases to a design task of $40 \mathrm{~h}$ design time (i.e. 1 working week) being completed over the course of 4 weeks. A fragment of a typical calculation sheet is shown in Fig. 3.

\begin{tabular}{|c|c|c|c|c|c|c|c|}
\hline QSB Bolometer & & & & & & & \\
\hline Average reekly design time [h] & 33 & $\operatorname{Jan} 11$ & Feb 11 & Mar 11 & Apr 11 & May 11 & Jun \\
\hline Planned Value $[\mathrm{h}]$ & PV & 1815 & 1947 & 2112 & 2244 & 2376 & 254 \\
\hline Earned Value $[\mathrm{h}]$ & EV & 1416 & 1562 & 1720 & 1820 & 1948 & \\
\hline Actual Cost [h cumulated] & AC & 1462 & 1606 & 1812 & 1914 & 2065 & \\
\hline Actual Cost [h current] & AC & 142 & 144 & 206 & 102 & 151 & \\
\hline Completion in \% & & $32 \%$ & $35 \%$ & $38 \%$ & $41 \%$ & $43 \%$ & \\
\hline Planned Value [h current] & PV & 132 & 132 & 165 & 132 & 132 & 16 \\
\hline Budget at Completion [h] & BAC & 4000 & 4480 & 4480 & 4480 & 4480 & \\
\hline Remaining budget [h as from EV] & BAC & 2584 & 2918 & 2760 & 2660 & 2532 & \\
\hline Remaining budget [h as from $\mathrm{AC}]$ & BAC & 2538 & 2874 & 2668 & 2566 & 2415 & \\
\hline Design AEV21 bolometer camera & 1160 & $96 \%$ & $96 \%$ & $97 \%$ & $97 \%$ & $97 \%$ & 97: \\
\hline AEV21 sightline aligning \& proving system & 80 & $0 \%$ & $0 \%$ & $0 \%$ & $0 \%$ & $0 \%$ & 0 \\
\hline Design Line of Sight Calibration System (HBC and VBC) & 40 & $0 \%$ & $0 \%$ & $0 \%$ & $0 \%$ & $0 \%$ & 0 \\
\hline Design AEU30 Bolometer Camera & 800 & $8 \%$ & $11 \%$ & $13 \%$ & $13 \%$ & $13 \%$ & 13: \\
\hline Manuf. dravings AEV21 bolometer camera & 720 & $11 \%$ & $21 \%$ & $38 \%$ & $50 \%$ & $67 \%$ & 67: \\
\hline Manufacturing Dravings Bolometer AEU30 & 720 & $5 \%$ & $8 \%$ & $8 \%$ & $8 \%$ & $8 \%$ & 8 \\
\hline Assembly and Design of the HBC & 320 & $0 \%$ & $0 \%$ & $0 \%$ & $0 \%$ & $0 \%$ & 0 \\
\hline ECRH test AEV 218 AEU 30 & 320 & $0 \%$ & $0 \%$ & $0 \%$ & $0 \%$ & $0 \%$ & 0 \\
\hline Space reservation AEV21 first iteration & 80 & $75 \%$ & $88 \%$ & $100 \%$ & $100 \%$ & $100 \%$ & 100 \\
\hline Space reservation AEV21 complete finished & 80 & $0 \%$ & $0 \%$ & $5 \%$ & $20 \%$ & $20 \%$ & $20:$ \\
\hline
\end{tabular}

Figure 3. Fragment of EVM calculation sheet (in MS Excel format). Only layer of main work packages and monthly data is shown, basic data containing all work packages and weekly PV and AC values are updated via embedded links.

\section{Results}

EVM data charts of engineering processes for 2 diagnostics are shown in Figures 4 and 5. The EV and AC graphs in both figures reflect a typical schedule and cost performance as observed on a number of diagnostics. EV curves fall behind schedule soon after the start of the monitoring; however, they keep the required gradient of the PV curve. In some cases they even catch up the PV lost at the start (Fig.4). This result fits well to the observations of a temporary progress slowdown during the transition process when the EVM infrastructure in the various W7-X departments has been established.

Another outcome, also characteristic of most diagnostic projects, is the slowly increasing gap between PV and AC as seen in Fig.5. This means that designers working on these diagnostics do not regularly reach the design time of $33 \mathrm{~h}$ per week estimated at the beginning of monitoring process in

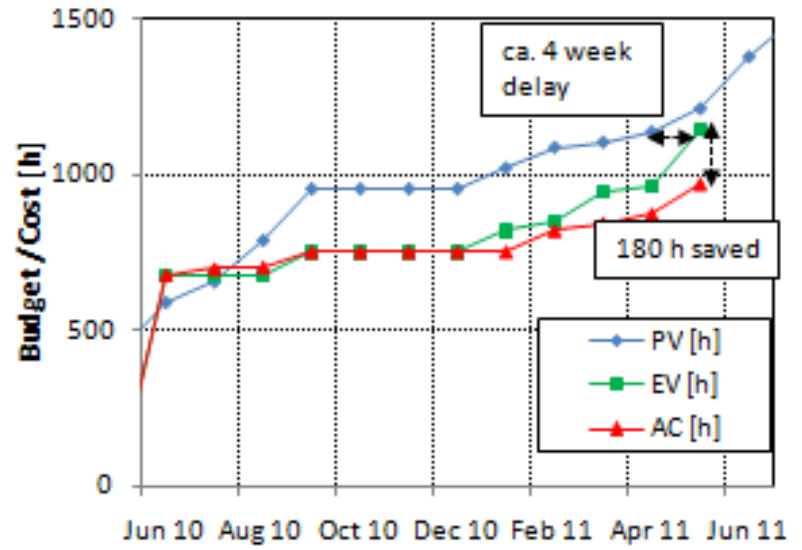

Figure 4. EVM chart for engineering of $\mathrm{H} \alpha$ diagnostic. PV stagnates due to a temporary reallocation of design resource following a decision by management between Sep 2010 and Jan 2011.

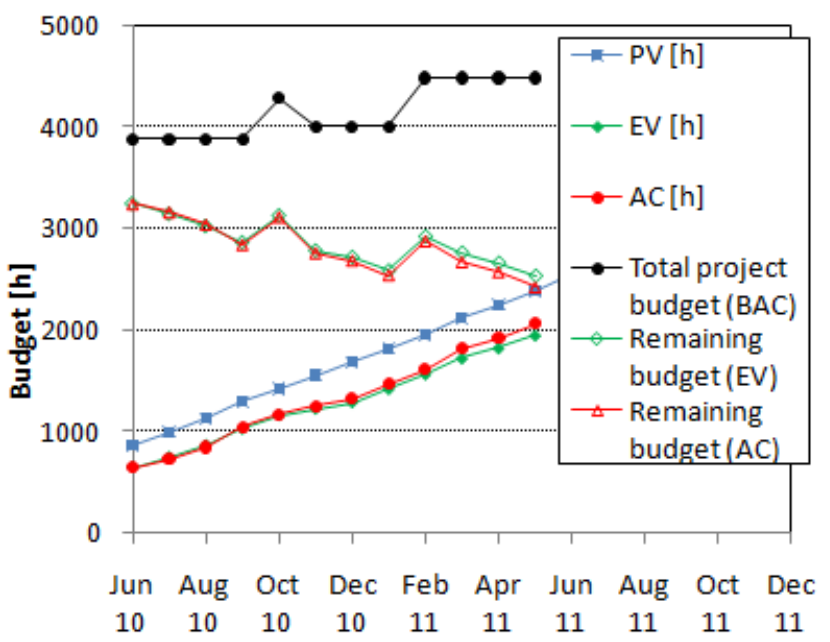

Figure 5. EVM chart for engineering of bolometer diagnostic with additional information on evolution of total (BAC) and remaining budgets. Remaining budgets have been assessed based on both, progress per EV and per AC. 
January 2010. Since this observation has been made on the majority of diagnostic projects analysis and optimization of internal management processes has been started. Amongst obvious issues, such as the number of internal meetings, the involvement in the decision process for a technical problem requires optimization. In some cases, the $33 \mathrm{~h}$ design time per week has been adapted to be within reach of AC leading to new, i.e. more realistic delivery milestones for design and manufacturing drawings of the diagnostics concerned.

A monthly, interdepartmental management meeting has been implemented along with the EVM to discuss EVM performance of each diagnostic and to decide on required changes. Typical changes include the temporary stagnation of PV - as seen in Fig. 4 - when the design had to be interrupted because the respective designer had to be reallocated to another diagnostic with higher time criticality.

Another example of change management is shown in Fig.5, where classical EVM performance indicators are shown together with information on the evolution of total (BAC) and remaining design budgets. Calculating the remaining budgets, both on EV and on AC performance allows for a robust completion time estimate. Fig.5 also illustrates how the BAC can evolve during the design of a single diagnostic. In this case a noticeable BAC increase had to be inserted in Feb. 2011, after a number of changes were requested in a design review.

The described simple handling of budget and work content changes in this tool turns out to be essential as during the engineering process many diagnostics are prone to requiring changes, partly due to unforeseen testing, but mostly due to analyzing and detailing the design packages into more standardized tasks. Thus, monitoring of a diagnostic using EVM benefits most, when the start of the design work was caught and when the design tasks are well understood by the customer (e.g. diagnostician). The latter also helps to avoid repeated and cumbersome harmonization of the often different planning "languages" of WBS and EVM.

In addition to the EVM output this diagnostic tool has been equipped with a milestone monitoring system. In fact, a weakness of the EVM method is circumvented. If design on one urgently needed work package hits unexpected technical issues which cannot be resolved by the designer, he is able to switch to another work package, the completion of which is required much later according to the WBS. Completion of this package, however, will lead to awarding EV (as the method suggests), although the most urgent work package has actually fallen behind schedule.

Comparing the required delivery milestones of each engineering package and the achieved progress according to the EVM in a suitable diagram gives a fast and transparent status check. Fig. 6 shows such a monitoring chart for the engineering milestones of the $\mathrm{H}_{\alpha}$ diagnostic. Each pair of progress data and completion milestones is shown in one format to separate the pairs from each other more easily. The milestone (deadline) “Typ II detailed design” has been completed almost on time;

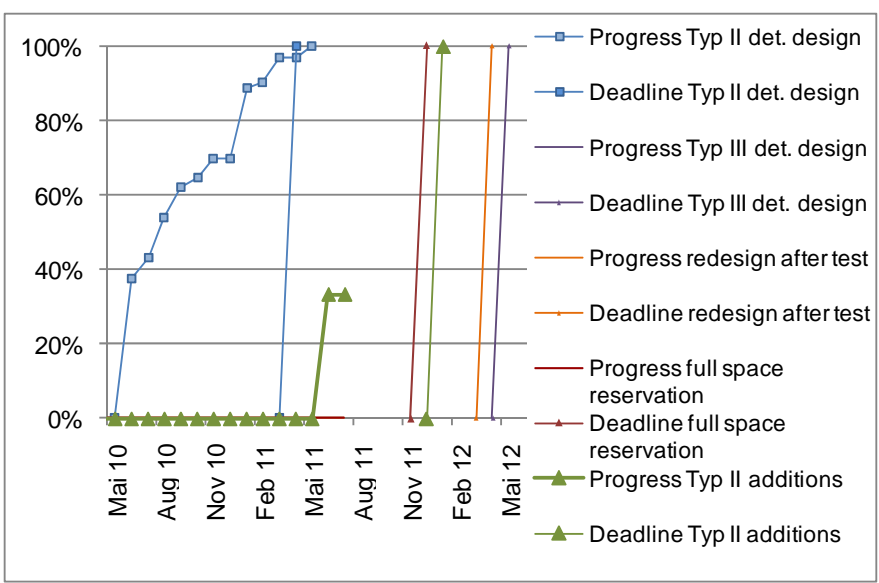

Figure 6. Monitoring chart for engineering milestones (deadlines) of $\mathrm{H} \alpha$ diagnostic. 5 pairs of progress lines and deadlines are shown grouped by colour. Each progress line was deduced from EVM chart and each completion deadline was deduced from WBS.

now attention is focusing on progress in packages "Type II additions" and "Full space reservation". Although the delay on “Typ II detailed design” was small, the implications were being monitored permanently and checked against buffers and requirement dates for drawing handover in line with manufacturing and assembly dates.

Regarding the monitoring of engineering milestones, the interdepartmental management meeting has a second mission: to decide on switching designers from one diagnostic to another, if technical, schedule, resource or even budgetary constraints require. A first lesson from applying this tool has been to ensure an efficient handover of information during such a "stop and go" phase in the design process.

\section{EVM TOOL FOR IN-VESSEL COMPONENT MANUFACTURING}

\section{A. Goal}

EVM has also been introduced to measure performance of the small series fabrication (up to $200 \mathrm{pc}$ ) of the in-vessel components (IVC) of W7-X, among them baffles, heat shields, wall panels, divertor target elements and cooling circuit plugins. In spite of their often complex geometric shapes to fit the 3D environment of W7-X, fabrication of the IVC has been organized as small batch production consisting of specialized, but reproducible manufacturing processes. Cost control is one of the monitoring goals.

\section{B. Set-Up}

The IVC are being manufactured and assembled mostly by internal resources in internal workshops on specialized lines (machining, brazing, welding, assembling, vacuum testing) on the basis of manufacturing orders. Since some components have been under fabrication for a number of years, the EVM system was organized to handle both, new and ongoing manufacturing. Accurate synchronization between the scope of the manufacturing orders and the existing WBS 
planning for each component was the key to finding the work packages, i.e. to determining PV and EV. Obviously, when catching the manufacturing start of a new component, this synchronization was part of the set-up. In this EVM tool, allocation of AC benefited enormously from the existing IPP administrative system (SAP) producing for each manufacturing order monthly reports with accurate recording of the internal and external hours worked.

\section{Results}

Fig. 7 shows the EVM graphs of the plug-ins for in-vessel cooling water supply as an example of a set of components just beginning fabrication. Since monthly EV and AC data availability is still being optimized, the steady rise of PV is followed by "nervous" EV and AC trajectories. In this phase, EVM monitoring emphasis rests on cost containment and predictions for the expected budget at completion, since production planning for the new component contains a number of assumptions on resources and fabrication rates. Supported by the accurate AC data collection, first results show that EV does not reach the level of AC, i.e. the planned budget might not suffice once all plug-ins will be completed. Since production of the plug-ins is to be completed by the end of 2011, countermeasures are being discussed with the responsible department.

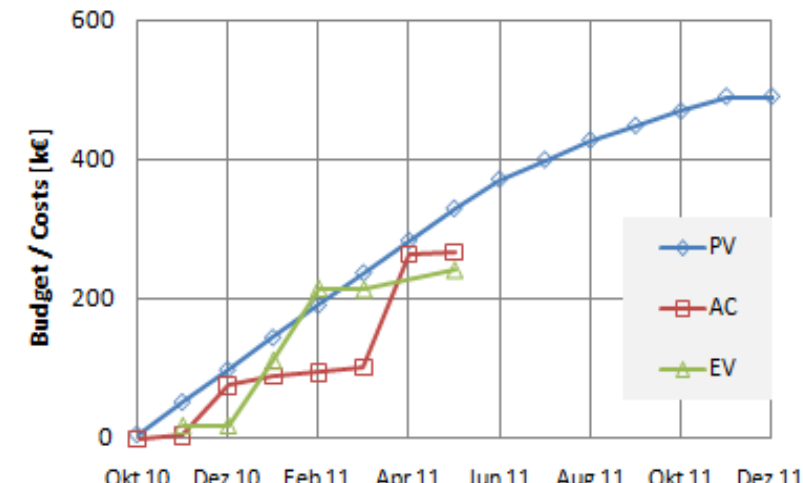

Figure 7. EVM chart for fabrication of plug-ins for cooling water supply.

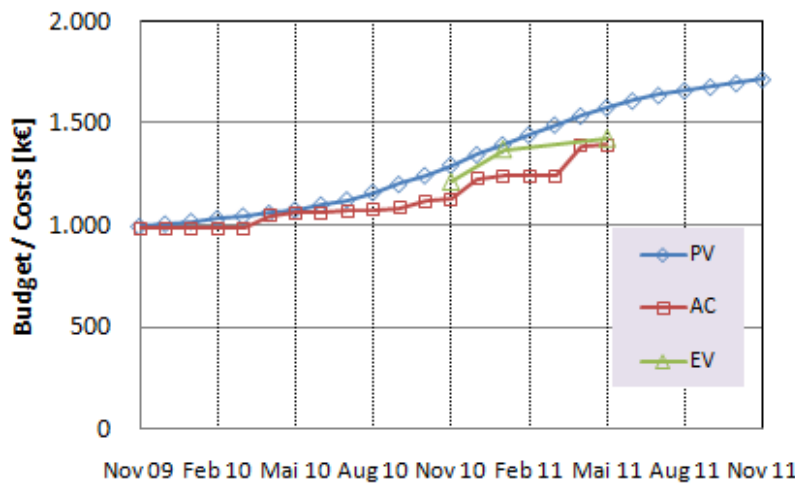

Figure 8. EVM chart for baffle production (running since 2006), notable EV measurement only in Nov 2010.
An example of ongoing IVC production is presented in Fig. 8 with baffle fabrication running since 2006. Since manufacturing priority was switched to other IVC in 2010, the gradient of the PV curve remained expectedly flat. EV measurement was introduced to catch the start of a reinforced baffle production phase in 2011, again cost containment being the main driver. Early results are encouraging, which can be seen by AC matching EV albeit with some schedule delay.

\section{SUMMARY}

Realization of EVM in W7-X has posed several challenges characteristic of large scale research projects. Since their respective implementation, all tools serve the department of Project Control as an early and transparent performance indicator focused on cost overruns and schedule delays. Nevertheless, a number of lessons have been learned during set-up and application of the tools.

- Breakdown of a baseline schedule into well defined, accountable and reliable work packages to determine PV and EV is particularly difficult in surroundings of numerous technical changes. Naturally, the handling of these changes has to be carefully built into the tool already during its set-up.

- $\quad$ Another major task is the adaptation of the cost analysis to specific boundary conditions of the project organization in order to correlate the costs as correctly as possible to the respective work packages. Especially, in very complex projects, e.g. the Wendelstein 7-X assembly, the desired level of detail of AC has to be balanced carefully against the effort required for a detailed allocation of the costs to the respective work package. The concept of the EVM tool, i.e. the adaptation of the method into the constraints of the in-house organization has to be detailed sufficiently before implementation. Extra work can be avoided, if the decision on data integration from several sources (often unavoidable) and on the software package to be used for the tool, are prepared carefully.

- Implementation of an EVM tool at the start of a new project avoids a number of limitations determining the performance and accuracy of the method when introduced later, i.e. in ongoing projects.

- $\quad$ However, EVM must not be used as the only monitoring tool on a given process since its time resolution is limited. In W7-X a time resolution of one month has been adopted for most parameters due to the effort related to collecting and analyzing data for $\mathrm{EV}$ and $\mathrm{AC}$.

EVM has proven to be an extremely versatile and even flexible tool as often non-transparent processes such as engineering and design of diagnostics and in-house manufacturing of complex components have been given a clearly structured monitoring system. The overriding prerequisite for efficient daily operation of the tools remains, however, a disciplined data exchange (data quality, data delivery), and accurate and effective communication across departments of the enterprise. 


\section{ACKNOWLEDGMENT}

The authors wish to thank the W7-X Division Heads, L. Wegener (Assembly) D. Hartmann (Design \& Configuration), the Department Head R. Stadler (IVC) and the W7-X Project Control Team for their contributions.

\section{REFERENCES}

[1] Project Management Institute, "Practice Standard for Earned Value Management”, Philadelphia, 2005.

[2] T. Bräuer et al., "Progress, Challenges and Lessons Learned in the Construction of Wendelstein 7-X", to be published in IEEE Trans. Plasma Science. 